\title{
Changes in Clinical and Histological Characteristics of Nasal Polyps in Northern China over the Past 2-3 Decades
}

\author{
Jiaqi Yu ${ }^{a}$ Mu Xian $^{a}$ Yingshi Piao $^{\mathrm{b}} \quad$ Luo Zhang $^{\mathrm{a}, \mathrm{c}, \mathrm{d}, \mathrm{e}} \quad$ Chengshuo Wang ${ }^{\mathrm{a}, \mathrm{c}, \mathrm{e}}$ \\ aDepartment of Otolaryngology, Head and Neck Surgery, Beijing TongRen Hospital, Capital Medical University, \\ Beijing, China; 'bepartment of Pathology, Beijing TongRen Hospital, Capital Medical University, Beijing, China; \\ 'Beijing Key Laboratory of Nasal Diseases, Beijing Institute of Otolaryngology, Beijing, China; ${ }^{\mathrm{d} D e p a r t m e n t}$ of \\ Allergy, Beijing TongRen Hospital, Capital Medical University, Beijing, China; ${ }^{~ R e s e a r c h ~ U n i t ~ o f ~ D i a g n o s i s ~ a n d ~}$ \\ Treatment of Chronic Nasal Diseases, Chinese Academy of Medical Sciences, Beijing, China
}

\section{Keywords}

Asthma $\cdot$ Chronic rhinosinusitis with nasal polyps · Inflammatory cell type · Phenotype · Polyp recurrence

\begin{abstract}
Introduction: Recent studies have shown that inflammatory patterns of nasal polyps from patients with chronic rhinosinusitis (CRS) with nasal polyps (CRSwNP) in East Asia have changed over time. However, to date there is a marked lack of similar data for CRSwNP in Northern China. This study thus aimed to assess the changes in the clinical and histological characteristics of CRSwNP patients from Northern China over the past 2-3 decades. Methods: This was a retrospective study, which examined data from 2 groups of 150 CRSwNP patients each, who had undergone endoscopic sinus surgery in Beijing Tongren Hospital from 1993 to 1995 (group A) and from 2015 to 2019 (group B). All relevant data for demographic, clinical, and histological parameters were collected for each patient from the 2 groups and compared for overall changes between the 2 groups. Results: The comorbidity of CRSwNP and asthma increased over time and the cellular phenotype of CRSwNPchanged significantly; in
\end{abstract}

karger@karger.com www.karger.com/iaa

Karger $\stackrel{\text { ' }}{=}$
(C) 2021 The Author(s)

Published by S. Karger AG, Basel

This is an Open Access article licensed under the Creative Commons Attribution-NonCommercial-4.0 International License (CC BY-NC) (http://www.karger.com/Services/OpenAccessLicense), applicable to the online version of the article only. Usage and distribution for commercial purposes requires written permission. particular, the proportion of eosinophil-dominant CRSwNP increased, lymphocyte-dominant and plasma-dominant CRSwNP decreased significantly, and the proportions of neutrophil-dominant and mixed CRSwNP were not altered. The rate of polyp recurrence increased in CRSwNP but did not in eosinophilic CRSwNP. Smoking and age did not significantly impact the inflammatory patterns of CRSwNP. Conclusions: The inflammatory patterns of CRSwNP patients have changed and comorbidity of asthma significantly increased in CRSwNP patients in Northern China over the past 2-3 decades.

(c) 2021 The Author(s)

Published by S. Karger AG, Basel

\section{Introduction}

Chronic rhinosinusitis (CRS) is a condition characterized by mucosal inflammation of the nose and paranasal sinuses and often classified as CRS without nasal

Jiaqi Yu and Mu Xian contributed equally to the study. Edited by: H.-U. Simon, Bern. 
polyps or CRS with nasal polyps (CRSwNP) based on the absence or presence of polyps. CRSwNP is a heterogeneous sinonasal inflammatory disease affecting approximately $2.1-4.4 \%$ of the Western population [1-3] and $1.1-2.7 \%$ of the Asian population [4, 5]. Clinically, CRSwNP can further be classified into eosinophilic CRSwNP (ECRSwNP) and noneosinophilic CRSwNP based on the presence of predominant inflammatory cells [6]. We have previously demonstrated that CRSwNP patients can be clustered into 5 phenotypes with different polyp recurrence rates based on the presence of predominantly plasma cells, lymphocytes, neutrophils, eosinophils, or mixed inflammatory cells [7]. The presence of tissue eosinophilia in CRSwNP is frequently associated with severe sinus disease, more incidence of comorbid asthma, and a higher polyp recurrence rate [7-9]. In contrast, patients with plasma cell-dominant and lymphocyte-dominant phenotypes show milder symptoms, are less likely to report comorbid asthma, and have lower recurrence rates [7].

Although identification of the inflammatory patterns of CRSwNP is likely to be helpful in selection of better treatment strategies and predicting the outcome, the inflammatory patterns of CRSwNP exhibit significant geographic and ethnic differences and may have changed over time. In this regard, studies have demonstrated that CRSwNP patients in Asia show lower eosinophilic and greater neutrophilic inflammation than CRSwNP patients in Western countries $[6,10]$. Recently, several studies have reported that eosinophilic inflammation has been significantly augmented over time in Asia [11-15]. However, most of these studies focused on the changes in eosinophilic inflammation, and none investigated either the changes in the other cellular phenotypes of CRSwNP or polyp recurrence.

CRSwNP is associated with a variety of clinical factors, with asthma as an important comorbid condition. It has been shown that CRSwNP is significantly associated with adult-onset asthma (onset after 18 years) or late-onset asthma (onset after 40 years) [16]. On the other hand, age is a major determinant for the incidence and clinical presentations of CRSwNP [17]. Similarly, cigarette smoking and exposure to passive smoke have been associated with CRS [18]. To date, there is little information on the effect of comorbid asthma, age, and smoking on the inflammatory pattern of CRSwNP over time. In view of this paucity, our study aimed to assess any changes in the clinical and pathological characteristics of CRSwNP in patients from Northern China over a period of 22 years.

\section{Materials and Methods}

\section{Subjects}

This was a retrospective, single-center study employing data collected from 2 groups of 150 CRSwNP patients each, who had undergone endoscopic sinus surgery after failure of medical treatment, in Beijing Tongren Hospital from December 1993 to May 1995 (group A) and from November 2015 to January 2019 (group B), respectively. Most of the patients (279/300) were from Northern China, with 145 patients of group A coming from 8 major cities and 134 patients of group B coming from 12 major cities in the north (Fig. 1). All patients had satisfied the diagnostic criteria for CRSwNP, as recommended by the European Position Paper on Rhinosinusitis and Nasal Polyps [10], and none had been treated with antibiotics or oral corticosteroids within the 4 -week period before surgery. The patients suffering from fungal rhinosinusitis, antrochoanal polyps, cystic fibrosis, or primary ciliary dyskinesia had been excluded from the study. Recurrence of polyps was characterized as the presence of diseased mucosa (nasal polyps, mucopurulent secretions, and/or inflamed mucosa) by nasal endoscopy, together with bothersome symptoms that persisted for at least 1 month and the need for maximal medical treatment [19]. Patients from 2015 to 2019 were followed up in a clinic with endoscopic examination, with a mean follow-up period of $35.8 \pm 12.1$ months and at least 18 months. Similarly, patients from 1993 to 1995 were also followed up in a clinic, with a mean follow-up time of $36.8 \pm$ 8.1 months and at least 26 months. Allergy was confirmed based on a positive skin prick test to a panel of common allergens or positive serum antigen-specific IgE, measured by the ImmunoCAP 100 system (Pharmacia, Uppsala, Sweden). Asthma was confirmed based on history and physician diagnosis. Smoking was confirmed based on history (at least 1 cigarette per day for at least 6 months and smoked within the last month). Elderly patients were defined as those aged 60 years or older. This study was approved by the Medical Ethics Committee of Beijing Tongren Hospital, and written informed consent was waived because of the retrospective nature of the study.

\section{Histological Evaluation}

Polyp tissues were obtained from endoscopic surgery and were fixed in $10 \%$ formaldehyde overnight at $4^{\circ} \mathrm{C}$, prior to processing for staining with hematoxylin and eosin $(\mathrm{H} \& \mathrm{E})$. The H\&E-stained specimens were obtained from the pathology department of the hospital and were observed by 2 independent pathologists, who were blinded to the clinical records of all patients. The numbers of neutrophils, eosinophils, plasma cells, lymphocytes, and total inflammatory cells in the lamina propria were counted in 10 nonoverlapping random fields at $\times 400$ magnification using a brightfield light microscope (BX51; Olympus, Japan) and recorded as the mean of 10 fields. In case of disagreement, when the 2 observers' counts differed by $>10 \%$, a consensus was reached by several members of the research team reviewing the specimen at the same time using a multi-head microscope. All specimens were then divided into 5 subtypes, as previously described [7], including eosinophildominant (eosinophil proportion $\geq 54.5 \%$ ), lymphocyte-dominant (lymphocyte proportion $\geq 64 \%$ ), neutrophil-dominant (neutrophil proportion $\geq 29 \%$, lymphocyte proportion $<64 \%$, and eosinophil proportion $<30 \%$ ), plasma cell-dominant (plasma cell proportion $\geq 20 \%$, neutrophil proportion $<29 \%$, lymphocyte proportion $<64 \%$, and eosinophil proportion $<30 \%$ ), and mixed inflammation 
Fig. 1. Distribution of cities in Northern China where the patients came from. Patients from 1993 to 1995 (a). Patients from 2015 to 2019 (b). The blue and green areas cover all the cities in northern China, and the red dots indicate the cities where the patients came from.

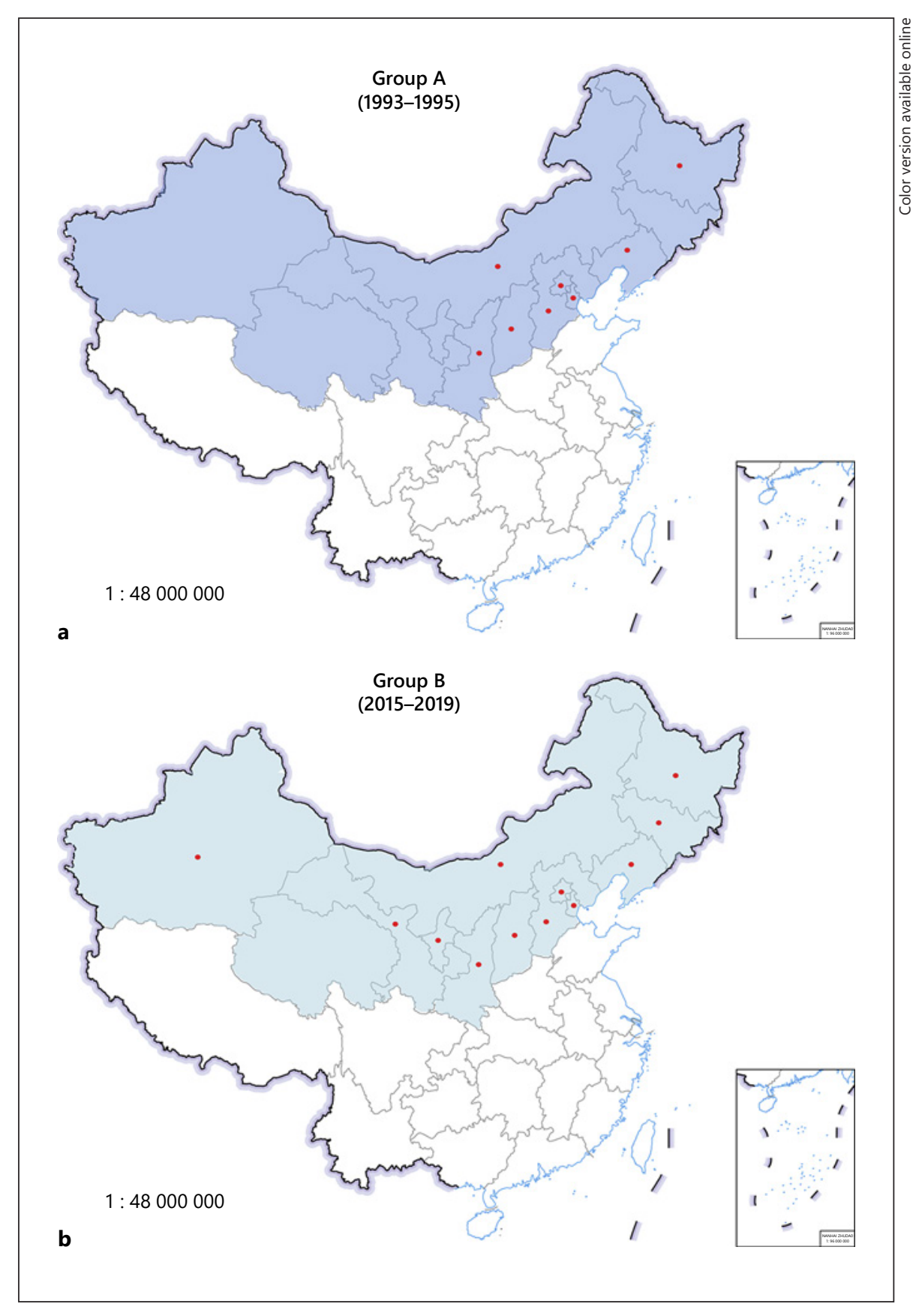

(lymphocyte proportion <64\%, 30\% < eosinophil proportion $\leq 54.5 \%$ or eosinophil proportion $<30 \%$, lymphocyte proportion $<64 \%$, plasma cell proportion $<20 \%$, and neutrophil proportion $<29 \%)$ phenotypes. The basement membrane thickness was measured for 5 fields of each section at $\times 400$ magnification, and the mean linear length was calculated [20]. The severity of stromal edema was evaluated and scored semi-quantitatively on a scale of $0-3$ according to the percentage of the stromal area observed under low-power magnification, with $0=$ no stroma, $1=<1 / 3,2=1 / 3$ to $2 / 3$, and $3=>2 / 3$ of the stromal area.

Changes in Characteristics of Nasal Polyps over Time

\section{Statistical Analysis}

For normally distributed continuous variables, the results were expressed as mean $\pm \mathrm{SD}$, and for nonnormally distributed continuous variables, the results were expressed as medians and interquartile ranges. The $t$ test and Mann-Whitney test were used for intergroup comparisons, and differences in proportions between groups were analyzed using the $\chi^{2}$ test. The Spearman test was performed for correlation analyses. All statistical analyses were performed using SPSS and GraphPad Prism. A value of $p<0.05$ was considered significant. 


\section{Results}

\section{Demographic and Clinical Characteristics Analysis}

Demographic and clinical characteristics of both groups of patients are shown in online suppl. Table 1; see www.karger.com/doi/10.1159/000513312 for all online suppl. materials. The patients in group B were slightly older than those in group A $(46.0 \pm 11.5$ vs. $42.8 \pm 14.5$, $p=0.037)$. The age of the onset of chronic sinusitis in group B was also higher than that in group A ( $37.5 \pm 12.4$ vs. $31.6 \pm 14.5, p<0.001)$. Similarly, the rate of comorbid asthma in group B was higher than in group A (35.3 vs. $2 \%, p<0.001)$, as was the rate of polyp recurrence within 3 years after surgery ( 55.3 vs. $34.7 \%, p=0.002)$. In contrast, the number of smokers in group $B$ was lower than that in group A ( 21.3 vs. $34.7 \%, p=0.010)$. However, the 2 groups were not significantly different with regard to sex distribution, the rate of comorbid allergic rhinitis, or previous endoscopic surgical history.

\section{Analysis of Histological Features}

Assessment of inflammatory cells in nasal polyps demonstrated that samples from patients in group B generally had a significantly higher number of eosinophils and total inflammatory cells than samples from patients in group A (eosinophils: median $=69.0 /$ high-power field $(\mathrm{HPF})$ vs. $16.0 / \mathrm{HPF}$; total inflammatory cells: median $=$ 227.5/HPF vs. 190.5/HPF). In contrast, the number of plasma cells and lymphocytes was significantly lower in samples from group B than group A, and there was no significant difference in the number of neutrophils between the 2 groups (Table 1). Assessment of different cells types as percentage of the total inflammatory cells confirmed that eosinophils were significantly higher and plasma cells and lymphocytes were significantly lower in samples from patients in group B than samples from group A. Assessment of the basement membrane in samples from group B further demonstrated that this was significantly thicker than that in samples from group A; however, the degree of tissue edema was comparable between the 2 groups (Table 1).

\section{Analysis of Cellular Phenotype}

In order to further explore the changes in inflammatory patterns of CRSwNP patients, we classified and compared the cellular phenotypes of patients in the 2 groups, according to the findings of Lou and colleagues [7] (Fig. 2). We found that the 2 groups were significantly

Table 1. Histological characteristics of nasal polyps from CRSwNP patients in group A and group B

\begin{tabular}{lcc}
\hline Histological parameter, median (IQR) & Group A: 1993-1995 $(n=150)$ & Group B: 2015-2019 $(n=150)$ \\
\hline Eosinophil count/HPF & $16.0(4.0-55.3)$ & $69.0(17.8-160.0)^{* * *}$ \\
Neutrophil count/HPF & $0.0(0.0-4.0)$ & $0.0(0.0-9.3)$ \\
Lymphocyte count/HPF & $103.0(67.0-159.0)$ & $78.5(30.8-150.0)^{* *}$ \\
Plasma cell count/HPF & $28.5(19.0-52.25)$ & $20.0(10.0-40.0)^{* * *}$ \\
Total inflammatory cell count/HPF & $190.5(137.8-261.3)$ & $227.5(124.3-375.0)^{*}$ \\
Tissue eosinophils, \% & $8.0(2.3-31.1)$ & $36.6(10.4-64.9)^{* * *}$ \\
Tissue neutrophils, \% & $0.0(0.0-2.4)$ & $0.0(0.0-3.0)$ \\
Tissue lymphocytes, \% & $59.4(44.6-72.8)$ & $42.1(19.9-63.7)^{* * *}$ \\
Tissue plasma cells, \% & $17.5(9.2-28.4)$ & $9.5(4.5-21.0)^{* * *}$ \\
BM thickness, $\mu \mathrm{m}$ & $3.9(2.6-6.1)$ & $8.7(5.2-13.3)^{* * *}$ \\
Tissue edema score & $1(0-2)$ & $1(0-2)$ \\
\hline
\end{tabular}

HPF, high-power field; IQR, interquartile range; CRSwNP, chronic rhinosinusitis with nasal polyp; BM, basement membrane. ${ }^{*} p<0.05$. ${ }^{* *} p<0.01 .{ }^{* * *} p<0.001$.

Fig. 2. Changes of cellular phenotype. a Comparison of the proportion of 5 cellular phenotypes in group A and group B. b-f Comparison of the proportion of eosinophil-dominant and non-eosinophil-dominant phenotypes, mixed inflammation and nonmixed inflammation phenotypes, neutrophil-dominant and nonneutrophil-dominant phenotypes, lymphocyte-dominant and nonlymphocyte-dominant phenotypes, and plasma cell-dominant and non-plasma cell-dominant phenotypes between the 2 groups. Group A, polyps obtained in 1993-1995; group B, polyps obtained in 2015-2019. $p$ value for comparisons between group A and group B. ${ }^{*} p<0.05 ;{ }^{* *} p<0.01$; $* * * p<0.001$.

(For figure see next page.) 


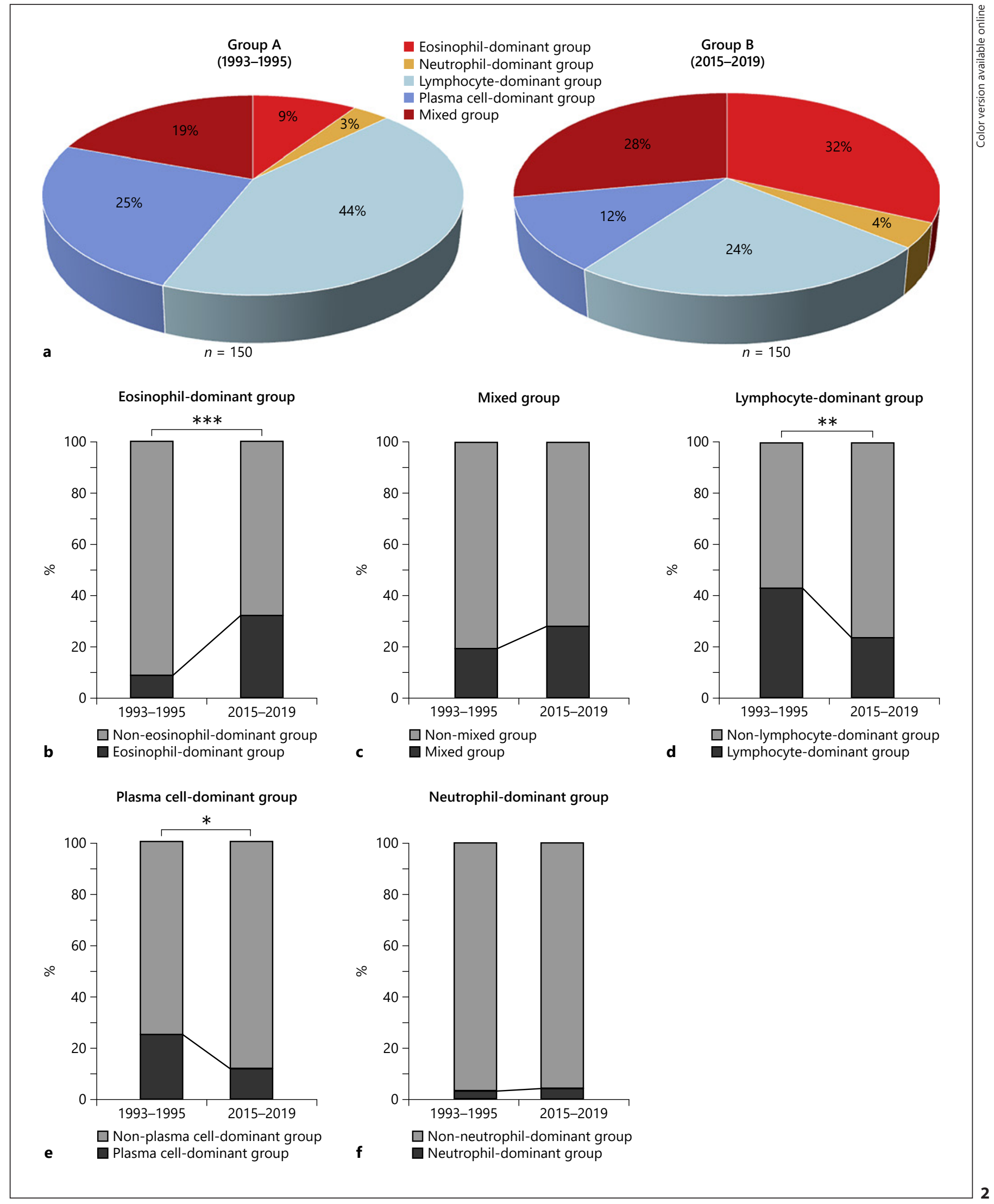

Changes in Characteristics of Nasal Polyps over Time

Int Arch Allergy Immunol 2021;182:615-624 
Fig. 3. Accuracy of predicting polyp recurrence according to tissue eosinophilia in different groups of CRSwNP patients. a Accuracy of predicting polyp recurrence according to the absolute tissue eosinophil count $>55 /$ HPF. b Accuracy of predicting polyp recurrence according to $>27 \%$ tissue eosinophilia. HPF, high-power field; CRSwNP, chronic rhinosinusitis with nasal polyp.

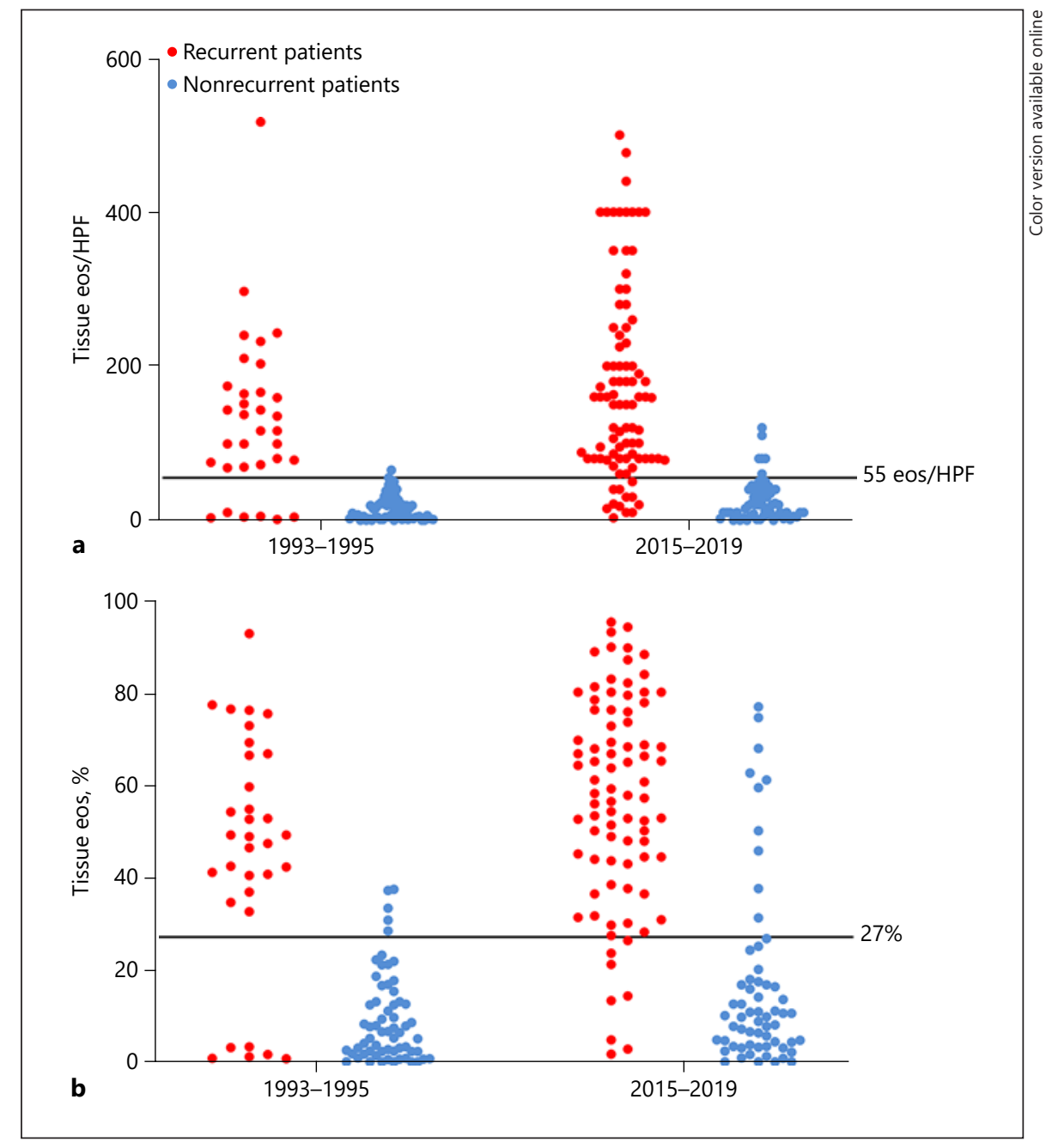

different in this respect (Fig. 2a, $p<0.001$ ). In particular, the proportion of patients with an eosinophilic-dominant phenotype was significantly higher in group $B$ than group A ( 32.0 vs. $9.3 \%, p<0.001$; Fig. $2 \mathrm{~b}$ ), and the proportions of patients with the lymphocyte-dominant phenotype (Fig. $2 \mathrm{~d}, p=0.007$ ) and plasma-dominant phenotype (Fig. 2e, $p=0.028$ ) were significantly lower in group $\mathrm{B}$ than group A. Although the proportion of patients with a mixed inflammation phenotype was also found to be higher in group B than group A, the difference was not statistically significant ( 28.0 vs. $19.3 \%, p=0.182$; Fig. 2 c). Similarly, the proportion of patients with neutrophildominant phenotype was not significantly different between the 2 groups (Fig. 2f, $p=0.702$ ).

As the eosinophil-dominant phenotype is known to be associated with higher asthma morbidity and recurrence within 2 years, we compared the demographic and clinical characteristics of patients with this cellular phenotype in the 2 groups. This assessment demonstrated that comorbid asthma was significantly higher in eosinophil-dominant patients from group B than eosinophil-dominant patients from group A; however, there was no significant difference in any other demographic and clinical characteristics between the 2 groups (online suppl. Table 2).

\section{Accuracy of Predicting Polyp Recurrence by Tissue Eosinophilia in Different Periods}

We have previously shown that a percentage of tissue eosinophils $>27 \%$ or a tissue eosinophil count $>55 / \mathrm{HPF}$ is an effective predictor of polyp recurrence within 2 years [19]. We investigated whether this criterion applied to patients with nasal polyps from group A (1993-1995). Employing a value of $27 \%$ for tissue eosinophils as the standard, the Youden index, sensitivity, and specificity 
for recurrence were estimated to be $73.8,81.8$, and $91.9 \%$, respectively (online suppl. Table 3 ). Similarly, employing an absolute tissue eosinophil count of 55/HPF yielded a Youden index of $80.2 \%$, with $81.8 \%$ sensitivity and $98.4 \%$ specificity.

Assessment of the tissue eosinophils in samples from patients in group B demonstrated that a value of $27 \%$ tissue eosinophils yielded the Youden index, sensitivity, and specificity values for recurrence of $73.5,89.9$, and $83.6 \%$, respectively; and an absolute tissue eosinophil count of $55 / \mathrm{HPF}$ yielded a Youden index, sensitivity, and specificity values for recurrence of $76.7,86.5,90.2 \%$, respectively (online suppl. Table 3). Both parameters of tissue eosinophilia exhibited good predictive values in different periods, and 55/HPF showed a better predictive value (Fig. 3; online suppl. Table 3 ).

\section{Impact of Smoking and Age}

Online suppl. Table 4 shows the effect of smoking on the clinical and pathological characteristics of CRSwNP patients in the 2 groups. Overall, both groups had twice as many male smokers as female smokers. Although the number and percentage of eosinophils were higher in the tissue of smokers than in non-smokers in both groups, the difference was only significant for the patients in group A. Furthermore, the smokers in group B had fewer complications of asthma and milder edema than nonsmokers in the same group.

Assessment of any correlation between age and the inflammation in CRSwNP demonstrated that age was not associated with either the absolute tissue eosinophil count/HPF (online suppl. Fig. 1a, age vs. eosinophils/ HPF in group $\mathrm{A}, R=0.036, p=0.666$; age vs. eosinophils/ HPF in group $\mathrm{B}, R=0.025, p=0.762$ ), or absolute tissue neutrophil count/HPF (online suppl. Fig. 1b, age vs. neutrophils/HPF in group $\mathrm{A}, R=-0.138, p=0.092$; age vs. neutrophils/HPF in group $\mathrm{B}, R=-0.048, p=0.560$ ). Even when the patients were stratified into 2 groups according to age $<60$ or $\geq 60 \mathrm{y}$, no difference was observed in the absolute tissue eosinophil and neutrophil count/HPF between the older and younger age-groups (online suppl. Fig. 1c, d).

\section{Discussion}

CRSwNP is a disease associated with a heavy social and economic burden [21]. As different subtypes of CRSwNP need different treatment strategies, it is important to explore the clinical and pathological features of patients for

Changes in Characteristics of Nasal

Polyps over Time optimum treatment. Our study aimed at assessing the overall changes in clinical and pathological characteristics of CRSwNP, which may have occurred over a longterm period of 22 years in Northern China and showed that eosinophilic inflammation in polyps has generally increased in Northern China. This is consistent with findings from other studies performed in Korea, Thailand, and China [11-15]. Employing 5 eosinophils/HPF as a diagnostic criterion for eosinophilia, Kim and colleagues [12] found that the proportion of ECRSwNP in Korea had increased from 24 to $51 \%$ over a period of 17 years. Similarly, Shin and colleagues [13] found that the incidence of ECRSwNP was significantly higher in 2011 (62.6\%) than in 2001 (52.3\%) and 2006 (47.7\%) in Korea. Studies by Katotomichelakis and colleagues [11] have indicated that eosinophilic inflammation also appeared to have increased in CRSwNP patients in Thailand, as shown by an increase in the median value from $5 / \mathrm{HPF}$ to $35 / \mathrm{HPF}$ over a period of 12 years. Moreover, one study by Jiang and colleagues [14] has shown that not only the proportion of ECRSwNP but also ECP+ eosinophils, IgE+ cells, and IL5+ cells were increased in CRSwNP patients from central China over a period of 14 years. Wang and colleagues [15] employed $>10 \%$ tissue eosinophils as a diagnostic criterion for eosinophilia and found that the proportion of ECRSwNP was significantly increased from 59.1 to $73.7 \%$ over a period of 11 years in Beijing, China. Although these studies have shown tissue eosinophilia to have increased in CRSwNP patients over the long term in several countries, the increase in eosinophilia has been defined employing relatively low cut-off values of 5 eosinophils/HPF or $10 \%$ eosinophils of total inflammatory cells in the tissue. In the present study, we have employed a much greater cut-off value of $>54.5 \%$ tissue eosinophils and confirmed that increased eosinophilic inflammation in nasal polyps in East Asia is likely to be a true reflection of the histopathological changes that have taken place in CRSwNP patients over the last 2-3 decades. However, the mechanisms underlying these histopathological changes are still unclear and a matter for further investigation in the future to better understand the etiology and management of this chronic condition.

Studies have reported that mucosal eosinophilia greatly increased the risk of recurrence in patients with nasal polyps [19,22]. In accordance with these studies, we found that there has also been an increase in the polyp recurrence rate, which is related to the remarkable increase in the ECRSwNP subtype over time. Furthermore, the present study has demonstrated that a tissue eosinophil proportion $>27 \%$ and an absolute tissue eosinophil count

Int Arch Allergy Immunol 2021;182:615-624 621 
over 55 eosinophils/HPF were good predictors of nasal polyp recurrence after surgery in both groups of patients, although this was found to be the case, especially in patients investigated from 1993 to 1995 . However, despite the advancement of functional endoscopic sinus surgery over the past decades, the rate of postoperative polyp recurrence in patients with ECRSwNP has not decreased sufficiently, suggesting that surgical treatment alone may not beenough for management of patients with ECRSwNP. Evidence from some studies that glucocorticoids are effective against eosinophilic polyps $[23,24]$ suggests that systematic application of glucocorticoids may be a suitable treatment option when eosinophils are noted in polyps of CRSwNP patients. However, short courses of oral corticosteroids may result in insomnia, mood and gastrointestinal disturbances [25], whereas continuous use of oral glucocorticoids lead to more serious side effects such as type 2 diabetes, gastric/duodenal ulceration, osteoporosis, and dyspeptic disorder [26]. In this regard, it has been suggested that biologics may offer a promising alternative for this group of CRSwNP patients [27].

The present study showed that there has been no significant change in neutrophil infiltration. Liao and colleagues [9] have demonstrated that a high proportion of CRSwNP patients with neutrophilic inflammation-dominated phenotype are difficult-to-treat CRS, suggesting that both eosinophilic and neutrophilic inflammation can lead to severe polyp disease. Although there is a crucial role for neutrophils in the pathophysiological processes of CRS, studies regarding the role of neutrophils in CRS are still sparse and at an early stage compared to the role of eosinophils, thus warranting greater attention in the future. The decreased infiltration of plasma cells in nasal polyps over time in this study was consistent with several relevant studies. Wang et al. [15] also found a significant decrease in the number of plasma cells in the polyp tissue over an 11-year period. Other studies also found a trend of decreasing plasma cells in the polyp tissue over years, although no significant difference was found [12, 14]. Meanwhile, a significant increase in the number of $\mathrm{IgE}+$ cells in the tissues was also found [14]. Although IgE-positive cells were predominantly mast cells in nasal polyps and there are few IgE-positive plasma cells, the source of IgE synthesis in the polyp tissues is plasma cells [28]. Gevaert et al. [29] reported that local class switch recombination to IgE was increased in CRSwNP in the European and B-cell differentiation into IgE-secreting plasma cells in NP. Moreover, Baba et al. [28] reported that the IgE concentration appears to be correlated with the magnitude of eosinophil infiltration in polyps, and compared to non-ECRS polyps, there was a decreased number of plasma cells and decreased expression of IgG mature transcripts in ECRS polyps. In the present study, we have also shown an increased number of eosinophils and a decreased number of plasma cells. Although we do not yet know the underlying mechanism, we speculate that it may be related to plasma cell isotype switching. A decrease in the number of lymphocytes was also observed over time, consistent with other studies $[12,14,15]$. In fact, due to the limitation of the technology 2-3 decades ago and the nature of the retrospective study, only H\&E staining was used in the present study, which was not effective in distinguishing the various subtypes of $\mathrm{T}$ cells and innate lymphocytes, both of which play a distinct role in CRS. Therefore, in future studies, we can specifically investigate the role of each subtype of immune cells by detecting cytokines or by flow cytometry. However, consistent with the findings of Jiang and colleagues [14] that there has been a marked increase in the thickness of the basement membrane in nasal polyps in adult CRSwNP patients in central China over the last few years, we also found that basement membrane in patients with CRSwNP had generally thickened over the last 2-3 decades. Indeed, the basement membrane thickness in CRS has been shown to be correlated with the number of infiltrating eosinophils and epithelial barrier damage [30].

To our knowledge, this is the first study to analyze and compare the change of the cellular phenotype of CRSwNP. We found that the trend of changes in the cellular phenotype of CRSwNP was consistent with the trend of changes in inflammatory cells in nasal polyp tissues, indicating that classification criteria set out by Lou and colleagues [7] for phenotyping CRSwNP are likely to be valuable in devising treatment strategies for CRS patients in the future. The reduction of plasma cell-dominant and lymphocyte-dominant polyps, which lack eosinophil and neutrophil infiltration, have a low recurrence rate (6$7 \%)$, and are less likely to report comorbid asthma and have mild symptoms [7], indicates a decrease in the proportion of nasal polyps with good prognosis. The lack of statistical difference in the proportion of mixed polyps may be due to the presence of other inflammatory cells, in particular the high increase in eosinophils in polyps. While the classification of the cellular phenotype of CRSwNP would help achieve accurate treatment of CRSwNP patients, the changes in the cellular phenotype of CRSwNP indicate toned for constant adjustment of a treatment plan to achieve a better outcome.

Asthma has been reported to be associated with CRS [16]. In the present study, we found that the comorbidity 
of CRSwNP and asthma has increased over time, especially in the eosinophilic subtype. This is in contrast to the findings of some previous studies, which showed no significant change in the comorbidity of CRSwNP and asthma $[11,14,15]$. While it is possible that this disparity is a consequence of differences in environmental factors and lifestyle [31], it is also possible this might be a consequence of patient bias resulting from the CRSwNP patients coming for treatment at a specialist clinic such as ours; that is, as CRSwNP with comorbid asthma is a complicated condition, patients with this complication have to visit tertiary class hospitals, which have specialists with the knowledge and skill to deal with the disease appropriately. Indeed, the complexity of CRSwNP and comorbid asthma being a more chronic and complicated condition than CRS without comorbid asthma has been further emphasized by a recent study of Novelli and colleagues [32] who demonstrated that asthmatics with CRSwNP had lower lung function and higher sputum eosinophilia than asthmatics without CRSwNP and that CRSwNP was an independent factor associated with airway eosinophilia in asthmatic patients.

Our study has some limitations. First, this was a retrospective study, and although data were collected in as much detail as possible, some data were not comparable for both groups of patients. For example, due to laboratory/technical limitations, the blood counts of inflammatory cells in CRSwNP patients and the data of CRSwNPrelated molecular marker such as IL-5 were not available in patients investigated from 1993 to 1995 . In addition, symptom score, CT score, and endoscopic score were not available for this group of patients; thus, it was difficult to compare the severity of disease between the 2 groups. Second, this was a single-center study, and the results were only applicable to patients from Northern China. Since CRSwNP shows significant regional differences, it is possible that patients from other regions and of different ethnicities may demonstrate different pathological features. Thus, multicenter national and international studies are required to assess the changes in the clinical and pathological features of CRS over the long term.

In conclusion, the comorbidity of CRSwNP and asthma has increased in Northern China over the last 2-3 decades, and the cellular phenotypes of patients with CRSwNP have changed significantly. In particular, the proportion of eosinophil-dominant phenotype of CRSwNP has increased substantially, while the proportions of lymphocyte-dominant and plasma-dominant phenotypes of CRSwNP have decreased significantly. In comparison, the proportions of neutrophil-dominant and mixed inflammation phenotypes of CRSwNP have not altered significantly. Moreover, smoking and age did not significantly impact the inflammatory patterns of CRSwNP. Although the recurrence rate of nasal polyps has generally increased in patients with CRSwNP, this has not changed in patients with ECRSwNP, likely as polyp recurrence was found to be associated with tissue eosinophilia. Nevertheless, as the pathophysiology of CRSwNP appears to have changed over the last 2 to 3 decades, treatment strategies for management of CRSwNP patients need to be adjusted accordingly and reviewed regularly in the future.

\section{Acknowledgement}

The authors would like to thank Yang Wang and Ying Li for their assistance in experimental procedures.

\section{Statement of Ethics}

This study was approved by the Medical Ethics Committee of Beijing Tongren Hospital, and written informed consent was waived because of the retrospective nature of the study.

\section{Conflict of Interest Statement}

The authors have no conflicts of interest to declare.

\section{Funding Sources}

This work was supported by grants from the National Key R\&D Program of China (2018YFC0116800, 2016YFC0905200), the Program for the Changjiang Scholars and Innovative Research Team (IRT13082), the National Natural Science Foundation of China (81630023, 81870698, 81500771), Beijing Municipal Administration of Hospitals' Mission Plan (SML20150203), Beijing Municipal Administration of Hospitals' Dengfeng Plan (DFL20190202), Beijing Municipal Administration of Hospitals Clinical Medicine Development of Special Funding Support (XMLX201816), and CAMS Innovation Fund for Medical Sciences (2019-I2M-5-022).

\section{Author Contributions}

All authors participated in the drafting/revision of the manuscript, final approval of the manuscript, and interpretation of findings. J.Y. and M.X. contributed to most of the experiments, including study design, study implementation, data analysis, and manuscript drafting. Y.P. participated in parts of experimental procedures. L.Z. and C.W. were involved in the design of the study and revision of the manuscript. All authors agreed to be accountable for all aspects of the work. 


\section{References}

1 Settipane GA, Chafee FH. Nasal polyps in asthma and rhinitis. A review of 6,037 patients. J Allergy Clin Immunol. 1977;59(1): $17-21$.

2 Hedman J, Kaprio J, Poussa T, Nieminen MM. Prevalence of asthma, aspirin intolerance, nasal polyposis and chronic obstructive pulmonary disease in a population-based study. Int J Epidemiol. 1999;28(4):717-22.

3 Klossek JM, Neukirch F, Pribil C, Jankowski R, Serrano E, Chanal I, et al. Prevalence of nasal polyposis in France: a cross-sectional, case-control study. Allergy. 2005;60(2):2337.

4 Shi JB, Fu QL, Zhang H, Cheng L, Wang YJ, Zhu DD, et al. Epidemiology of chronic rhinosinusitis: results from a cross-sectional survey in seven Chinese cities. Allergy. 2015; 70(5):533-9.

5 Ahn JC, Kim JW, Lee CH, Rhee CS. Prevalence and risk factors of chronic rhinosinusitus, allergic rhinitis, and nasal septal deviation: results of the Korean National Health and Nutrition Survey 2008-2012. JAMA Otolaryngol Head Neck Surg. 2016;142(2):162-7.

6 Cao PP, Li HB, Wang BF, Wang SB, You XJ, Cui $\mathrm{YH}$, et al. Distinct immunopathologic characteristics of various types of chronic rhinosinusitis in adult Chinese. J Allergy Clin Immunol. 2009;124(3):478-84, .

7 Lou H, Meng Y, Piao Y, Zhang N, Bachert C, Wang C, et al. Cellular phenotyping of chronic rhinosinusitis with nasal polyps. Rhinology. 2016;54(2):150-9.

8 Tomassen P, Vandeplas G, Van Zele T, Cardell LO, Arebro J, Olze H, et al. Inflammatory endotypes of chronic rhinosinusitis based on cluster analysis of biomarkers. J Allergy Clin Immunol. 2016;137(5):1449-56.e4.

9 Liao B, Liu JX, Li ZY, Zhen Z, Cao PP, Yao Y, et al. Multidimensional endotypes of chronic rhinosinusitis and their association with treatment outcomes. Allergy. 2018;73(7): 1459-69.

10 Fokkens WJ, Lund VJ, Mullol J, Bachert C, Alobid I, Baroody F, et al. European position paper on rhinosinusitis and nasal polyps 2012. Rhinol Suppl. 2012;23:3 p preceding table of contents, 1-298.

11 Katotomichelakis M, Tantilipikorn P, Holtappels G, De Ruyck N, Feng L, Van Zele T, et al. Inflammatory patterns in upper airway disease in the same geographical area may change over time. Am J Rhinol Allergy. 2013; 27(5):354-60.
12 Kim SJ, Lee KH, Kim SW, Cho JS, Park YK, Shin SY. Changes in histological features of nasal polyps in a Korean population over a 17-year period. Otolaryngol Head Neck Surg. 2013;149(3):431-7.

13 Shin SH, Ye MK, Kim JK, Cho CH. Histological characteristics of chronic rhinosinusitis with nasal polyps: recent 10-year experience of a single center in Daegu, Korea. Am J Rhinol Allergy. 2014;28(2):95-8.

14 Jiang WX, Cao PP, Li ZY, Zhai GT, Liao B, Lu $\mathrm{X}$, et al. A retrospective study of changes of histopathology of nasal polyps in adult Chinese in central China. Rhinology. 2019;57(4): 261-7.

15 Wang W, Gao Y, Zhu Z, Zha Y, Wang X, Qi $\mathrm{F}$, et al. Changes in the clinical and histological characteristics of Chinese chronic rhinosinusitis with nasal polyps over 11 years. Int Forum Allergy Rhinol. 2019;9(2):149-57.

16 Won H-K, Kim Y-C, Kang M-G, Park H-K, Lee S-E, Kim M-H, et al. Age-related prevalence of chronic rhinosinusitis and nasal polyps and their relationships with asthma onset. Ann Allergy Asthma Immunol. 2018;120(4): 389-94.

17 Song WJ, Lee JH, Won HK, Bachert C. Chronic rhinosinusitis with nasal polyps in older adults: clinical presentation, pathophysiology, and comorbidity. Curr Allergy Asthma Rep. 2019;19(10):46.

18 Christensen DN, Franks ZG, McCrary HC, Saleh AA, Chang EH. A systematic review of the association between cigarette smoke exposure and chronic rhinosinusitis. Otolaryngol Head Neck Surg. 2018;158(5):801-16.

19 Lou H, Meng Y, Piao Y, Wang C, Zhang L, Bachert C. Predictive significance of tissue eosinophilia for nasal polyp recurrence in the Chinese population. Am J Rhinol Allergy. 2015;29(5):350-6.

20 Yan B, Wang Y, Li Y, Wang C, Zhang L. Inhibition of arachidonate 15-lipoxygenase reduces the epithelial-mesenchymal transition in eosinophilic chronic rhinosinusitis with nasal polyps. Int Forum Allergy Rhinol. 2019; 9(3):270-80.

21 Bhattacharyya N, Villeneuve S, Joish VN, Amand C, Mannent L, Amin N, et al. Cost burden and resource utilization in patients with chronic rhinosinusitis and nasal polyps. Laryngoscope. 2019;129(9):1969-75.
22 Wei B, Liu F, Zhang J, Liu Y, Du J, Liu S, et al. Multivariate analysis of inflammatory endotypes in recurrent nasal polyposis in a Chinese population. Rhinology. 2018;56(3):216-26.

23 Hong H, Wang D, Tan KS, Zheng R, Chen F, Gao W, et al. Sinus computed tomography predicts clinical response to corticosteroids in chronic rhinosinusitis with nasal polyps. Clin Transl Allergy. 2018;8:24.

24 Zhang Y, Wang C, Huang Y, Lou H, Zhang L. Efficacy of short-term systemic corticosteroid therapy in chronic rhinosinusitis with nasal polyps: a meta-analysis of randomized controlled trials and systematic review. Am J Rhinol Allergy. 2019;33(5):567-76.

25 Head K, Chong LY, Hopkins C, Philpott C, Schilder AG, Burton MJ. Short-course oral steroids as an adjunct therapy for chronic rhinosinusitis. Cochrane Database Syst Rev. 2016;4:Cd011992.

26 Karatzanis A, Chatzidakis A, Milioni A, Vlaminck S, Kawauchi H, Velegrakis S, et al. Contemporary Use of corticosteroids in rhinology. Curr Allergy Asthma Rep. 2017; 17(2):11.

27 Wang C, Zhang L. Use of biologics in chronic sinusitis with nasal polyps. Curr Opin Allergy Clin Immunol. 2019;19(4):365-72.

28 Baba S, Kondo K, Toma-Hirano M, Kanaya K, Suzukawa K, Ushio M, et al. Local increase in IgE and class switch recombination to IgE in nasal polyps in chronic rhinosinusitis. Clin Exp Allergy. 2014;44(5):701-12.

29 Gevaert P, Nouri-Aria KT, Wu H, Harper CE, Takhar P, Fear DJ, et al. Local receptor revision and class switching to $\operatorname{IgE}$ in chronic rhinosinusitis with nasal polyps. Allergy. 2013;68(1):55-63.

30 Bassiouni A, Ou J, Rajiv S, Cantero D, Vreugde S, Wormald PJ. Subepithelial inflammatory load and basement membrane thickening in refractory chronic rhinosinusitis with nasal polyposis: a histopathological study. Int Forum Allergy Rhinol. 2016;6(3): 248-55.

31 Paudel U, Pant KP. Beyond smoking: environmental determinants of asthma prevalence in Western Nepal. J Health Pollut. 2020; 10(25):200310.

32 Novelli F, Bacci E, Latorre M, Seccia V, Bartoli ML, Cianchetti S, et al. Comorbidities are associated with different features of severe asthma. Clin Mol Allergy. 2018;16:25. 